\title{
Efficient Floating Car Data Transmission via LTE for Travel Time Estimation of Vehicles
}

\author{
Christoph Ide, Brian Niehoefer \\ and Christian Wietfeld \\ Communication Networks Institute (CNI) \\ Faculty of Electrical Engineering and Information Technology \\ TU Dortmund University, Germany \\ Email: \{Christoph.Ide, Brian.Niehoefer, \\ Christian.Wietfeld $\} @$ tu-dortmund.de
}

\author{
Timo Knaup, Daniel Weber, \\ Lars Habel and Michael Schreckenberg \\ Physics of Transport and Traffic \\ University Duisburg-Essen, Germany \\ Email: $\{$ Knaup, Weber, Habel, \\ Schreckenberg\}@ptt.uni-due.de
}

\begin{abstract}
The travel time estimation of vehicles is a major challenge in the area of dynamic traffic prognosis. Our approach is to increase the number of considered sensor objects in the road network. For this purpose Floating Car Data (FCD) including travel time information of vehicles is transmitted to a server via Long Term Evolution (LTE). In this paper, the benefit of FCD on the accuracy of travel time estimation, depending on the FCD penetration rate is analyzed by an enhanced Nagel-Schreckenberg cellular automaton model. Furthermore, the negative impact of the FCD transmission on the air interface of the cellular communication system is evaluated for various penetration rates and different transmission strategies, including a channel sensitive transmission. Therefore, a close to reality parameterized Markovian model is used.

The results show that a penetration rate of a few percent is sufficient for a realistic travel time estimation. The respective influence on the LTE network is tolerable, especially for channel sensitive transmission.
\end{abstract}

\section{INTRODUCTION}

Due to the booming oil price and the declining traffic situation in many cities, innovative, high precision and real time traffic prognosis became a major research topic in the past years. Thereby, the accuracy of the prognosis depends on the quantity, quality and reliability of the available information within the traffic flow. Based on accurate travel time predictions, an optimal occupancy rate on the respectively used street segment can be realized by guiding individual vehicles on alternative routes, if necessary. The accuracy of the acquired data and a minimum delay of the data aggregation are essential to assure a useful outcome for the traffic prognosis.

Within the Collaborative Research Center SFB 876 we use automobile traffic prognosis as an example for an object flow optimization, with the aim to relieve temporary traffic hot spots created by accidents, car breakdowns or roadwork. In addition, the project is facing with an innovative data aggregation of the multitude of car-sensor data sources (for example GPS position, velocity and brake pressure), data fusion and communication methods with respect to the requirements set by the prognosis. Fig. 1 clarifies our approach. The use of Floating Car Data (FCD) for the traffic prognosis, especially the travel time estimation, and the according impact on the wireless cellular communication system (LTE) is analyzed. It is obvious, that the communication aspects of such approaches are as important as the actual prognosis. Transmitting additional traffic information is a minor priority service, compared to Internet access services using LTE technology (e.g. video streaming applications). Moreover, the characteristics of Vehicular to Infrastructure (V2I) traffic, which is part of Machine-to-Machine (M2M) communication, is different to conventional LTE Human-to-Human $(\mathrm{H} 2 \mathrm{H})$ communication [1], in relation to the transmitted amount of data and data rate. Hence, the negative impact on the existing infrastructure has to be as small as possible. To ensure this, we use a channel sensitive transmission for the FCD [2]. Furthermore, the benefit of the additional data for the prognosis decreases over time. So this paper deals with a comparative analysis of required FCD penetration rates and their benefit to microscopic traffic models, like the Nagel-Schreckenberg model [3], and with the influence to the LTE infrastructure which is analyzed by using a closed to reality Markovian model.

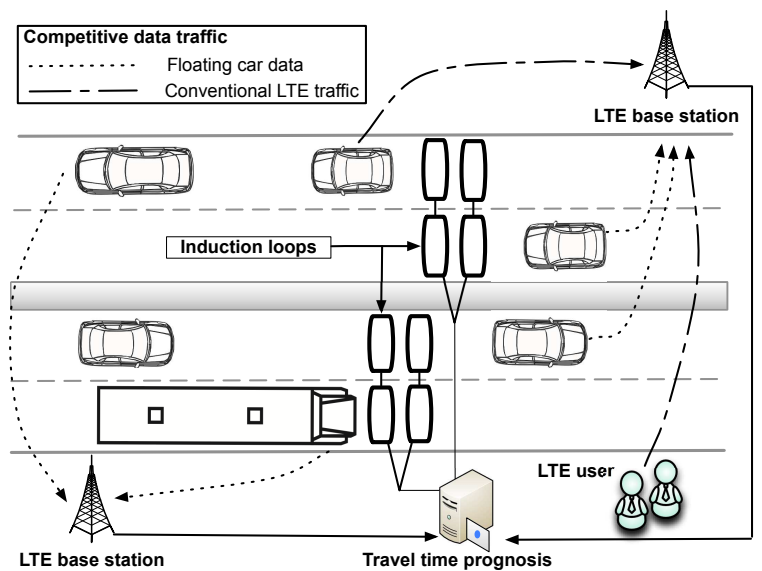

Fig. 1: Highway scenario with V2I communication via LTE for the transmission of Floating Car Data

The proceeding of this paper is organized as follows: After a short related works summary in Sec. II, the paper proceeds with a detailed description of the virtual FCD generation in 
Sec. III and a system overview of the used setup evaluating the influences on an LTE cell in Sec. IV. To point out the capabilities of our approach, Sec. V will depict the gained results for the necessary FCD penetration rate and the corresponding impact to the future LTE network. Finally, Sec. VI wraps up the key points in a conclusion.

\section{RELATED WORK}

In [4], FCD is used for the traffic state identification. Thereby, the quality of a small penetration rate of FCD vehicles for the reconstruction of the actual travel times is analyzed. In addition, the use of a micro simulation for FCD and their influence on wireless communication systems is shown in [5]. A study of necessary penetration rates based on traffic volume and arrival probabilities can be found in [6].

The inclusion of $\mathrm{M} 2 \mathrm{M}$ communication into conventional traffic of cellular communication systems is one of the main goals in the standardization process of LTE-Advanced [1], [7]. Hereby, the impact of hundreds of M2M devices on the normal $\mathrm{H} 2 \mathrm{H}$ communication should be as small as possible. Strategies to keep the complexity of M2M application on different layers as small as possible are shown in [8].

In [9] and [10] Markovian models are used to evaluate LTE networks. Thereby, the different states represent different channel characteristics. Markovian models for resource allocation, with one state representing a part of the shared resources, can be found in [11]. In [12] a multiclass Erlang loss model is introduced for Orthogonal Frequency-Division Multiplexing (OFDM) systems. Every state in the model represents one subcarrier. This Markovian model is adapted in this paper, to make it even more practice-oriented for LTE systems by using the Resource Blocks (RBs) as states. Furthermore, we parameterize the model with measurements and ray tracing simulations.

\section{TrafFic Simulator Generating Virtual FCD}

Our simulation is based on an enhanced NagelSchreckenberg model with velocity depended randomization [3], [13]. A one dimensional array of 1000 cells represents a single lane track, whereas a cell length of $7.5 \mathrm{~m}$ is used (see Fig. 2). Thereby, each cell can be occupied by at most one vehicle. The velocity $v$ of each vehicle is an integer with a value between 0 and $v_{m}$, in our case the maximum velocity $v_{m}$ is set to 4 .

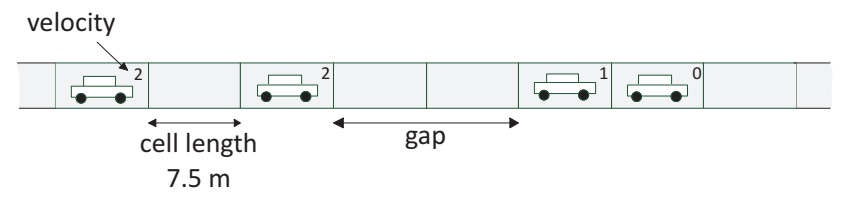

Fig. 2: Discrete vehicle positions according to the NagelSchreckenberg model for generating virtual FCD travel times

The update rules of the model are performed in parallel for all vehicles, with one time step set to 1 second. This corresponds to velocity bins of $27 \mathrm{~km} / \mathrm{h}$ and a maximal velocity $v_{m}$ of $108 \mathrm{~km} / \mathrm{h}$. Open boundary conditions and two bottleneck situations, one within the track (cells 500 to 550) and one at the end (last 4 cells) are used. If the leftmost cell of the array is empty, a vehicle will be inserted with a probability $\gamma$ and a velocity of $v_{m}$. If a vehicle is near the end of the track and the velocity is high enough to reach it in the next timestep, $\delta$ is the probability that it can leave the track. To simulate the bottlenecks within and at the end of the track the dawdling parameter $p$ is increased, respectively the parameter $\delta$ is smaller than $1(\delta=0.5)$. To realize the velocity depended randomization, the dawdling parameter $p$ is a function of the velocity (slow-to-start rule):

$$
p(v)=\left\{\begin{array}{ll}
0.5 & \text { for } v=0 \\
0.2 & \text { for } v>0
\end{array} .\right.
$$

In the bottleneck situation within the track, $p(v)$ is always set to 0.5 . In order to get a high flow situation, on average 1800 vehicles per hour are inserted onto the track and $\gamma=0.5$ is used.

After a sufficiently long relaxation time of 5000 time steps the simulation runs for further 15000 steps. Every vehicle which passes the track transmits its travel time as FCD. To evaluate the benefit of the upcoming prognosis, we simulate different penetration rates of FCD vehicles (5\%,1\% and $0.1 \%)$. Thereby, the corresponding vehicles are chosen randomly.

\section{SyStem MOdEL - LTE PERFORMANCE EVALUATION FOR FCD TRANSMISSION}

In order to evaluate the influence of the FCD transmission on $\mathrm{H} 2 \mathrm{H}$ communication, a system model consisting of laboratory measurements, ray tracing simulations and a Markovian model is used (see Fig. 3).

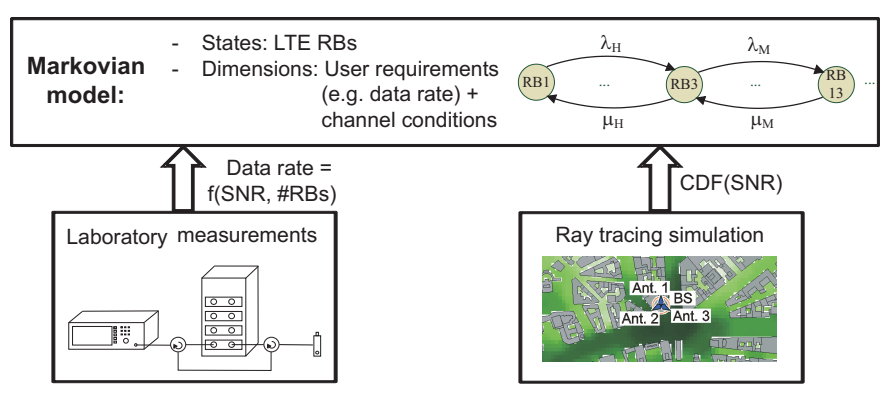

Fig. 3: System model for the evaluation of the influence of FCD transmission on the utilization of the LTE radio interface

Here, the behavior for many users in an LTE cell is described by a multidimensional Markovian model [14]. Assigning the user request to different classes of resources, each class is modeled by one dimension. We divide the users by their individual requirements $(250 \mathrm{kbit} / \mathrm{s}$ video streaming as typical $\mathrm{H} 2 \mathrm{H}$ application, number of Resource Blocks (RBs) are channel dependent; 1 kByte FCD UDP data, 10 RBs fixed), the Signal to Noise Ratio (SNR) and the user velocity. Hereby, the utilization of the LTE RBs is the bottleneck in our scenario. A detailed investigation of the signaling procedure and the 
random access, which are often critical in M2M scenarios is only necessary for thousands of devices in one cell [15]. In our case, the number of devices is much smaller, because the number of nodes is limited by the number of cars and the penetration rate. We use a typical urban highway scenario:

- $500 \mathrm{~m}$ base station radius

- One highway with 2x4 lines

- High traffic flow with one car per line every $20 \mathrm{~m}$

For this scenario and a penetration rate of $10 \%$ there are on average 40 device which send FCD per LTE cell.

\section{A. Laboratory Measurement Setup}

The Markovian model is parametrized by LTE data rate measurement results. For the evaluation of the UDP uplink data rate for different channel conditions, laboratory measurements are performed. A base station emulator set up a $10 \mathrm{MHz}$ LTE cell in the band $7(2.62 \mathrm{GHz})$. Hereby, the modulation and coding schemes with the index 0,3 and 6 and the acknowledged Radio Link Control (RLC) Automatic Repeat Request (ARQ) mode are used. The base station emulator is connected via RF cable to a fading channel emulator. On this equipment an ITU Vehicular A channel model [16] affects realistically the LTE signal. In addition, an Additive White Gaussian Noise (AWGN) with a fixed SNR can be added to the RF connection. The category 3 LTE User Equipment (UE) Samsung GT B3730 completes the setup. For the UDP data rate measurements the LTE base station and the UE are connected to two PCs with iPerf running. More details on the used setup, especially the channel emulator can be found in [17].

\section{B. SNR Estimation by Ray Tracing Simulations}

To evaluate the SNR for the urban scenario, we use a realistic channel model with a 3D Intelligent Ray Tracing (AWE Probsim [18]) and a detailed 3D CAD model of an urban scenario. This enables an accurate determination of the SNR. The base station antenna has an opening angle of $120^{\circ}$ with $16.7 \mathrm{~dB}$ gain and a downtilt of $3^{\circ}$. Furthermore, the UE antenna gain is $1 \mathrm{dBi}$, the UE noise figure is set to $6 \mathrm{~dB}$ and the transmission power of the UE is $23 \mathrm{dBm}$.

\section{Analytical Markovian Model}

For the evaluation of the performance of an LTE cell with many users and different QoS requirements, a Markovian model (multi-class Erlang loss model) is used [14]. In our model, the states represent the LTE RBs. Hence, the different traffic classes need a different number of states and each class is represented by a dimension in this model. According to the reduction of dimensions [19] a one dimensional model with a different number of states per user can be developed. The blocking probability for this model can be calculated for the assumption that the interarrival time for the arrival rate $\lambda_{s}$ and services rate $\mu_{s}$ for every class $s$ follows a negative exponential distribution.
According to [19] the stationary distribution $\pi_{c}$, which characterizes the probability that $c \mathrm{RBs}$ are allocated, can be determined reclusively

$$
\pi_{c}=\frac{\tilde{\pi}_{c}}{\sum_{c=0}^{C} \tilde{\pi}_{c}} \text { with } \quad \tilde{\pi}_{c}=\left\{\begin{array}{ll}
1 & c=0 \\
\sum_{s=1}^{S} \frac{a_{s} c_{s}}{c} \widetilde{\pi}_{c-c_{s}} & c>0
\end{array},\right.
$$

where $C$ is the maximum number of RBs used by an LTE base station (for $10 \mathrm{MHz}$ : 50), $a_{s}$ the offered traffic of class $s, c_{s}$ the resources of class $s$ and $S$ the number of service classes, i.e. dimension of the model. The blocking probability $p_{b_{s}}$ of $s$ and the overall traffic load $Y$ can now be calculated as

$$
p_{b_{s}}=\sum_{c=C-c_{s}+1}^{C} \pi_{c} \text { and } Y=\sum_{c=1}^{C} c \pi_{c} .
$$

The service rate for the $\mathrm{H} 2 \mathrm{H}$ communication is set to 1 per second and the service rate for the V2I users is calculated as data rate, which is taken from the laboratory measurements, divided by the transmitted FCD (1 kByte). Hereby, the ray tracing simulation defines the SNR for the data rate measurements.

\section{Channel Sensitive Transmission Scheme}

The FCD could be transmitted periodically. For this transmission strategy, the FCD is sent very often under bad channel conditions. Hence, we use a velocity and SNR sensitive FCD transmission [2]. The characteristic of the sending procedure is represented by an individual sending probability $p_{i, j}$ for every class of the Markovian model. The index $i$ is used for different SNRs and $j$ for different velocities.

$$
p_{i, j}=\frac{\left(\frac{S N R_{i}}{S N R_{\max }}\right)^{\alpha} \cdot\left(\frac{v_{\max }}{v_{j}}\right)^{\beta}}{\sum_{l=1}^{N} \sum_{k=1}^{M}\left(\frac{S N R_{l}}{S N R_{\max }}\right)^{\alpha} \cdot\left(\frac{v_{\max }}{v_{k}}\right)^{\beta}}, i=1 \ldots N, j=1 \ldots M
$$

Here, $S N R_{\max }$ is the SNR with the highest data rate and $v_{\max }$ is the highest assumed velocity. We use $S N R_{\max }=$ $40 \mathrm{~dB}$ and $v_{\max }=150 \mathrm{~km} / \mathrm{h}$. A method how to estimate the SNR in an OFDM system is explained in [20]. The parameters $\alpha$ and $\beta$ adjust the intensity of the channel sensitive transmission. For the Markovian model, we divided the SNR in $N=3$ parts and the velocity in $M=2$ parts. The values for the SNRs are estimated by the ray tracing simulation and the velocities $60 \mathrm{~km} / \mathrm{h}$ and $120 \mathrm{~km} / \mathrm{h}$ are used. The probability is included into the arrival rate of the different FCD classes of the Markovian model. The arrival rate for each class is multiplied with $p_{i, j}$.

\section{Results}

\section{A. Traffic Simulator}

To evaluate the necessary penetration rate of FCD vehicles providing their travel time to get a reliable traffic state estimation, a Monte Carlo Simulation is used. Fig. 4 
shows one representative result of the simulations out of 3000 iterations. In this simulation, we use $100 \%$ FCD penetration rate as reference. Thereby, every vehicle announces its travel time after passing the track. The obtained data is grouped into 5 minute intervals. To clarify the difference of varying penetration rates, the figure also illustrates the estimated travel time using $5 \%, 1 \%$ and $0.1 \%$ penetration rate of FCD vehicles in comparison to the ideal simulated travel time. If no FCD vehicle announced its travel time within a 5 minute interval the missing value is calculated by linear interpolation. The $5 \%$-curve is quite similar to the reference. It is obvious, that the shape of the $1 \%$-curve is still roughly the same but it diverges in some cases significantly. A estimation based on $0.1 \%$ FCD differs clearly from the ideal travel time. However, the results suggest, that a reliable travel time estimation with a few percent FCD vehicles should be possible.

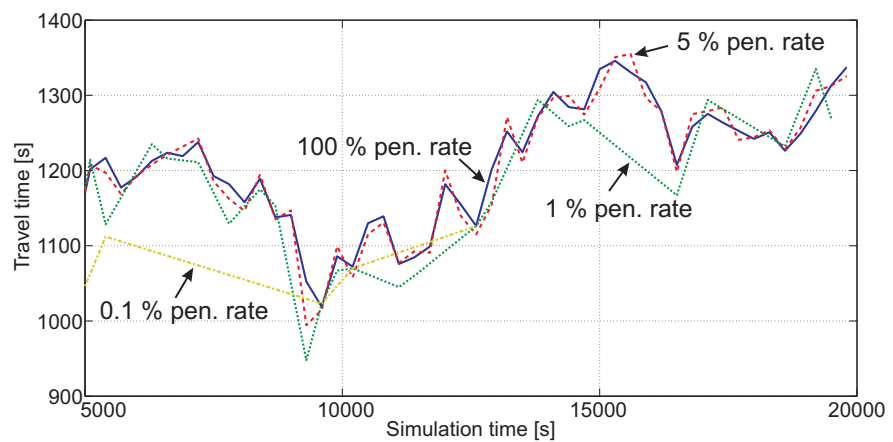

Fig. 4: Traffic simulator: Travel time estimation of total track for different FCD penetration rates

To validate these results, the empirical Cumulative Distribution Function (CDF) over all 3000 iterations illustrates the quality of the travel time estimation for the different penetration rates in the simulation. Therefore, the relative deviation between the travel times measured at $5 \%, 1 \%$ and $0.1 \%$ and the reference was calculated (see Fig. 5). It clearly indicates that a reliable travel time estimation with a minor FCD penetration rate is possible. In $90 \%$ the travel time deviation at $5 \%$ penetration rate is lower than $3.3 \%$, respectively $6 \%$ at a $1 \%$ penetration rate. That means, only a few percent penetration rate is sufficient for a reliable travel time estimation.

\section{B. Impact on the LTE System}

The UDP uplink data rates vs. SNR for different Modulation and Coding Schemes (MCS) are presented in Fig. 6. In order to determine the number of needed RBs to offer the data rate for the video streaming application, we also measured the data rate for other numbers of RBs. Hence, we know how many states in the Markovian model are required for different channel conditions.

In Fig. 7 the map of the urban scenario with the positionspecific SNR is illustrated. These results are taken from the ray tracing simulation. It can be seen that the SNR especially on the highway is very high.

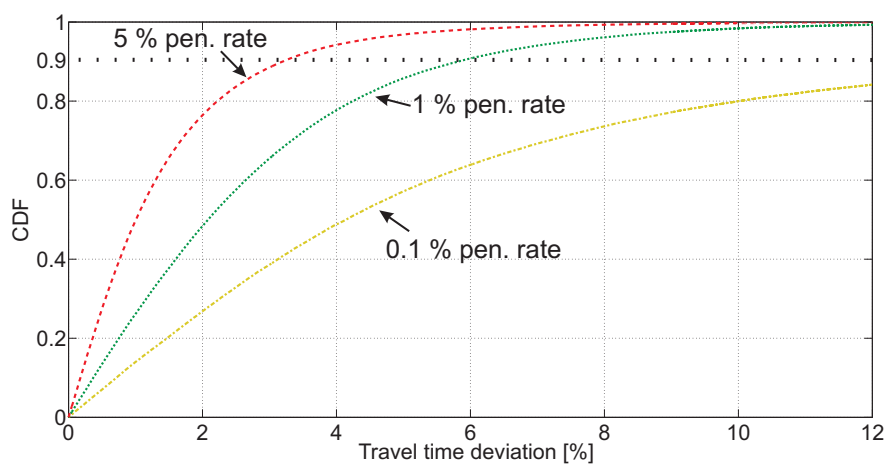

Fig. 5: Traffic simulator: $\mathrm{CDF}$ of the travel time deviation

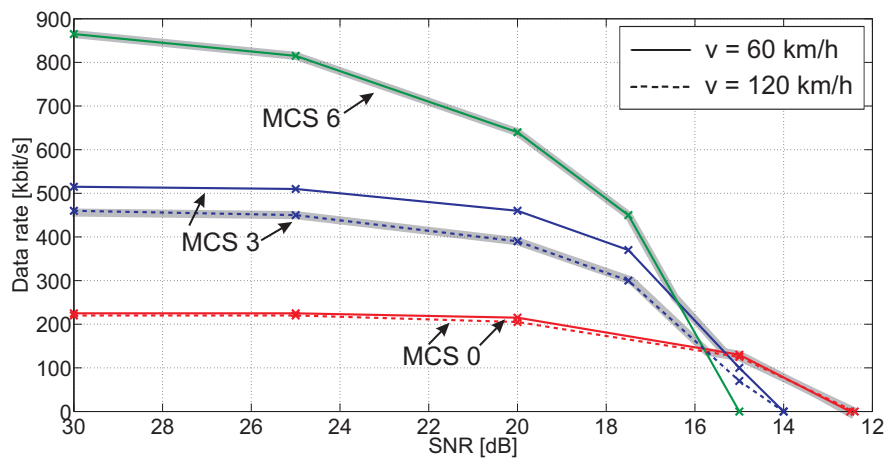

Fig. 6: Lab. measurements: Uplink data rate vs. SNR for 10 RBs per user under Vehicular A channel model

From the measurements, we identified that the impact of the SNR is stronger than the impact of the velocity on the data rate (see Fig. 6). Hence, we differentiated the users for the urban scenario according to the SNR into three fractions with the same size

- $\mathrm{SNR} \leq 17.5 \mathrm{~dB}$ : represented by $14 \mathrm{~dB}$ SNR

- $17.5 \mathrm{~dB}<\mathrm{SNR} \leq 26 \mathrm{~dB}$ : represented by $22 \mathrm{~dB}$ SNR

- $26 \mathrm{~dB}<$ SNR: represented by $30 \mathrm{~dB}$ SNR

and by the velocity into two parts

- $\mathrm{v} \leq 90 \mathrm{~km} / \mathrm{h}$ : represented by $60 \mathrm{~km} / \mathrm{h}$

- $90 \mathrm{~km} / \mathrm{h}<\mathrm{v}$ : represented by $120 \mathrm{~km} / \mathrm{h}$

The represented values are the weighted averages of the intervals.

Hence, for the parameters of the Markovian model the data rate for these three SNRs are taken from the laboratory measurements.

The results from the LTE performance analysis via the Markovian model can be found in Fig. 8. Here, the number of $\mathrm{H} 2 \mathrm{H}$ users, which can be served as a function of the number of V2I devices and the penetration rate is presented. For a typical QoS target of $10 \%$ blocking probability for the $\mathrm{H} 2 \mathrm{H}$ users $4 \mathrm{H} 2 \mathrm{H}$ video applications can be served per second for a FCD penetration rate of $5 \%$. By applying the channel sensitive transmission the number of $\mathrm{H} 2 \mathrm{H}$ applications can be increased to 6 per second. This is an enhancement of $50 \%$. If the $\mathrm{H} 2 \mathrm{H}$ users are alone in the LTE cell (arrival rate for M2M is equal to 0) 7 users can be handled. This means that the transmission 


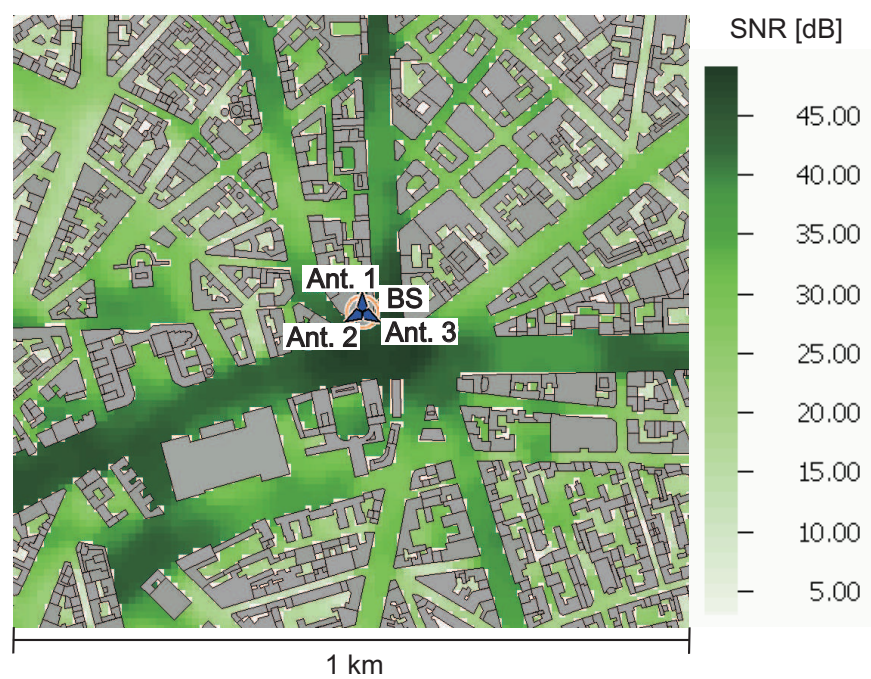

Fig. 7: Ray tracing: SNR results in the urban environment

of the FCD with channel sensitive transmission decreases the number of $\mathrm{H} 2 \mathrm{H}$ devices by only $17 \%$. For $1 \%$ penetration rate the decrement is only $4 \%$.

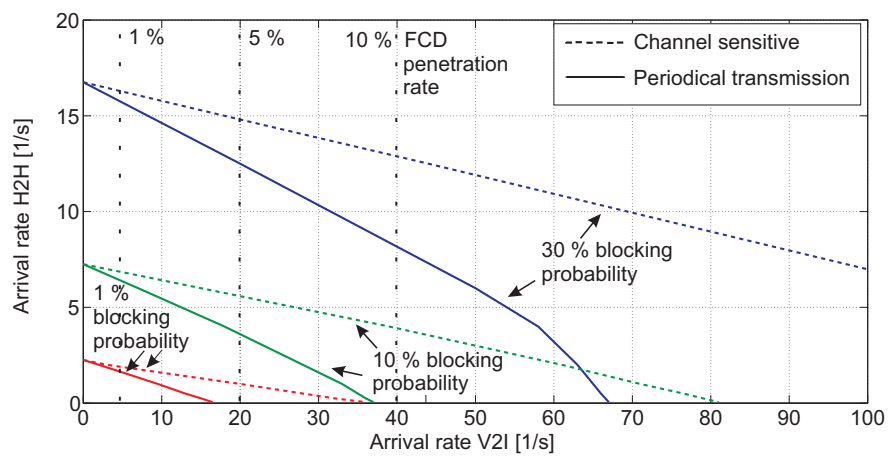

Fig. 8: Markovian model: Arrival rate of $\mathrm{H} 2 \mathrm{H}$ vs. arrival rate of V2I for different QoS requirements for the $\mathrm{H} 2 \mathrm{H}$ communication

\section{CONCLUSION}

In this paper, we analyzed the trade-off between the accuracy of the travel time estimation of vehicles and the negative impact on the LTE air interface. By using an extended Nagel-Schreckenberg model the required penetration rate for a reliable travel time prediction is estimated. Furthermore, a close to reality parameterized Markovian model is used to quantify the negative impact of the FCD transmission on the $\mathrm{H} 2 \mathrm{H}$ communication of the shared LTE network. We identified that only a few percent FCD penetration rate is needed for a significant travel time estimation.

By adopting our channel sensitive transmission approach, the negative impact of the FCD transmission on the number of servable human users per LTE cell decrease. Hence, the FCD can be transmitted without imposing noteworthy impact on the communication system.

\section{ACKNOWLEDGMENT}

Part of the work on this paper has been supported by Deutsche Forschungsgemeinschaft (DFG) within the Collaborative Research Center SFB 876 "Providing Information by Resource-Constrained Analysis", project B4 "Analysis and Communication for the Dynamic Traffic Prognosis".

\section{REFERENCES}

[1] 3GPP TS 22.368, Service Requirements for Machine-Type Communications, version 13.3

[2] C. Ide, B. Dusza, M. Putzke and C. Wietfeld, Channel Sensitive Transmission Scheme for V2I-based Floating Car Data Collection via LTE, IEEE International Conference on Communications (ICC) Workshop on Intelligent Vehicular Networking: V2V/V2I Communications and Applications, Ottawa, Canada, Jun. 2012

[3] K. Nagel and M. Schreckenberg, A Cellular Automaton Model for Freeway Traffic, J. Physique I 2, 22212229, 1992

[4] B. S. Kerner, C. Demir, R. G. Herrtwich, S. L. Klenov, H. Rehborn, M. Aleksi, A. Haug, Traffic state detection with floating car data in road networks, 8th International IEEE Conference on Intelligent Transportation Systems, Vienna, Austria, Sep. 2005

[5] B. S. Kerner, S. L. Klenov and A. Brakemeier, Testbed for Wireless Vehicle Communication: a Simulation Approach based on ThreePhase Traffic Theory IEEE Intelligent Vehicles Symposium, Eindhoven, Netherlands, Jun. 2008

[6] S. Breitenberger, B. Grueber, M. Neuherz and R. Kates, Extended Floating Car Data: Traffic Information Potential and necessary penetration rates, Traffic Eng. Contr., 45, (11), pp. 396-401, 2004

[7] 3GPP TR 23.888, System Improvements for Machine-Type Communications, version 1.2

[8] Y. Chen and W. Wang, Machine-to-Machine Communication in LTE$A$, 72st IEEE Vehicular Technology Conference, Ottawa, Canada, Sep. 2010

[9] F. Sallabi and K. Shuaib, Modeling of Downlink Wireless Fading Channel for 3GPP LTE Cellular System, IEEE GCC Conference and Exhibition, Dubai, United Arab Emirates, Feb. 2011

[10] J. She et al., Layered Adaptive Modulation and Coding for $4 G$ Wireless Networks, IEEE Global Telecommunications Conference, Miami, USA, Dec. 2010

[11] J. Ha, J.-U. Kim and S.-H. Kim, Cascaded Resource Allocation among Prioritized Shared Spectrum Blocks, 72st IEEE Vehicular Technology Conference, Ottawa, Sep. 2010

[12] V. Pla, J. Martinez-Bauset and V. Casares-Giner, Comments on "Call Blocking Probability and Bandwidth Utilization of OFDM Subcarrier Allocation in Next-generation Wireless Networks", Communications Letters, vol. 12, no. 5, May 2008

[13] R. Barlovic, L. Santen, A. Schadschneider and M. Schreckenberg, Metastable states in cellular automata for traffic flow, Eur. Phys. J. B 5, 793-800, 1998

[14] C. Ide, B. Dusza, M. Putzke, C. Mueller and C. Wietfeld, Influence of $M 2 M$ Communication on the Physical Resource Utilization of LTE, 11th Wireless Telecommunications Symposium, London, UK, Apr 2012

[15] ZTE, R2-104662: MTC Simulation Results with Specific Solutions, 3GPP TSG RAN WG2 Meeting 71, Aug. 2010

[16] International Telecommunication Union, Recommendation ITU-R M.1225 Guidelines for Evaluation of Radio Transmission Technologies for IMT-2000, 1997

[17] C. Ide, B. Dusza and C. Wietfeld, Mobile WiMAX Performance Measurements with Focus on Different QoS Targets, 18th IEEE Workshop on Local and Metropolitan Area Networks (LANMAN), Chapel Hill, USA, Oct. 2011

[18] G. Woelfle, R. Hoppe and F. M. Landstorfer, A Fast and Enhanced Ray Optical Propagation Model for Indoor and Urban Scenarios, Based on an Intelligent Preprocessing of the Database, 10th IEEE International Symposium on Personal, Indoor and Mobile Radio Communications, Osaka, Japan, Sep. 1999

[19] J. S. Kaufmann, Blocking in a Shared Resource Environment, IEEE Transactions on communications, vol. 29, no. 10, Oct. 1981

[20] S. A. Kim, D. G. An, H.-G. Ryu and J.-U. Kim, Efficient SNR estimation in OFDM system, IEEE Radio and Wireless Symposium (RWS), Phoenix, USA, Jan. 2009 